\title{
TOPS Interferometry With TerraSAR-X
}

\author{
Pau Prats-Iraola, Member, IEEE, Rolf Scheiber, Luca Marotti, \\ Steffen Wollstadt, and Andreas Reigber, Senior Member, IEEE
}

\begin{abstract}
This paper presents results on SAR interferometry for data acquired in the Terrain Observation by Progressive Scans (TOPS) imaging mode. The rationale to retrieve accurate interferometric products in this mode is expounded, emphasizing the critical step of coregistration. Due to the particularities of the TOPS mode, a high Doppler centroid is present at burst edges, demanding a very high azimuth coregistration performance. A coregistration accuracy of around one tenth of a pixel, as it is usually recommended for stripmap interferometric data, could result in large undesired azimuth phase ramps in each TOPS burst. This paper presents two approaches based on the spectral diversity technique to precisely estimate this coregistration offset with the required accuracy and evaluates their performance. The effect of squint at burst edges in terms of an undesired impulse response shift during focusing and the impact on the interferometric coregistration performance is also addressed. Repeat-pass TOPS data acquired experimentally by TerraSAR-X are used to validate the proposed approaches.
\end{abstract}

Index Terms-Coregistration, SAR interferometry, synthetic aperture radar (SAR), terrain observation by progressive scans (TOPS), TOPS interferometry.

\section{INTRODUCTION}

$\mathbf{T}$ ERRAIN Observation by Progressive Scans (TOPS) has been proposed as a new wide-swath imaging mode [1]. It overcomes the problems of scalloping and azimuth-varying signal-to-ambiguity ratio of the conventional ScanSAR mode by means of steering the antenna in the along-track direction. To achieve the same swath coverage and avoid the undesired effects of ScanSAR, the antenna is rotated throughout the acquisition from backward to forward at a constant rotation rate (see Fig. 1). The fast steering leads to a reduction of the target dwell time, and consequently a worsening of the azimuth resolution. However, as all targets are observed by the complete azimuth antenna pattern, the scalloping effect nearly eliminates, and the azimuth ambiguities and the signal-to-noise ratio become constant in azimuth. At the end of the burst, the antenna look angle is changed to illuminate a second subswath, pointing again backward. When the last subswath is imaged, the antenna points back to the first subswath, so that no gaps are left between bursts of the same subswath. The TOPS mode was first demonstrated in-orbit by TerraSAR-X [2], and it will be the default mode of operation of ESA's Sentinel-1 satellite [3].

Manuscript received April 12, 2011; revised September 21, 2011; accepted November 20, 2011. Date of publication January 13, 2012; date of current version July 18, 2012. This work was supported in part by the European Space Agency under contract 22243/09/NL/JA.

The authors are with the Microwaves and Radar Institute, German Aerospace Center (DLR), 82234 Wessling, Germany (e-mail: Pau.Prats@dlr.de; Rolf. Scheiber@dlr.de; Luca.Marotti@dlr.de; Steffen.Wollstadt@dlr.de; Andreas. Reigber@dlr.de).

Digital Object Identifier 10.1109/TGRS.2011.2178247

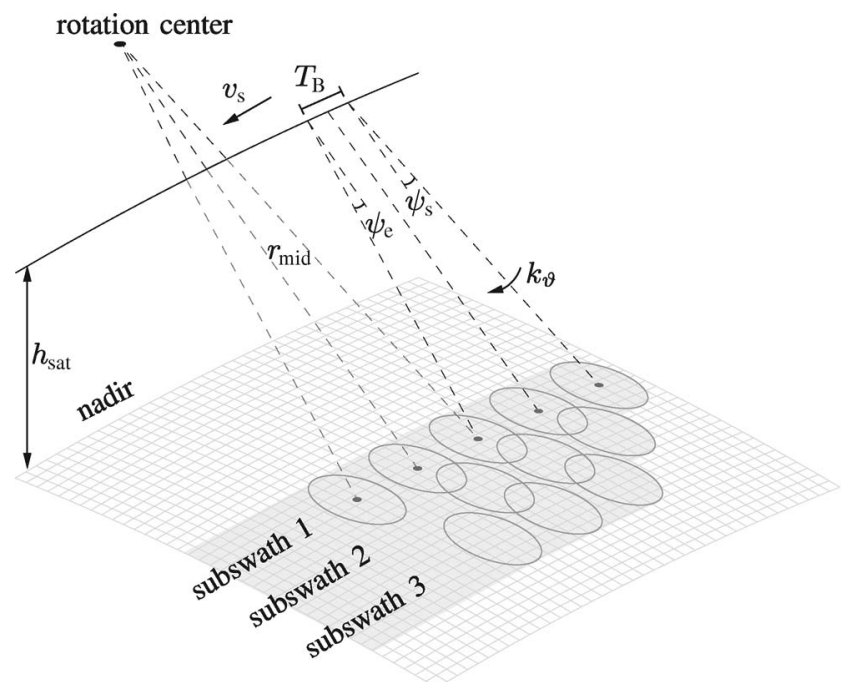

Fig. 1. Sketch of the TOPS acquisition geometry. $T_{B}$ is the burst duration, $r_{\text {mid }}$ is the midrange slant-range distance in a given subswath, $v_{s}$ is the satellite velocity, $h_{\text {sat }}$ is the satellite altitude, $k_{\vartheta}$ is the steering angle rate, and $\psi_{s}$ and $\psi_{e}$ are the starting and the end steering angles within the burst, respectively.

Similar to the ScanSAR mode, a precise pointing accuracy and along-track position knowledge are necessary in order to ensure an interferometric pair with overlapping azimuth spectra [1], [2]. However, one of the most challenging aspects in TOPS interferometry is the fact that the acquired data have large Doppler centroid variations within a burst. For typical TerraSAR-X TOPS acquisitions, the Doppler centroid can vary by more than $7 \mathrm{kHz}$ within each burst. It is well known that in the presence of squint, linear phase ramps are induced in the focused impulse response both in azimuth and range [4]-[6], where for small squints, the range phase ramp can be usually neglected [2].

Fig. 2 shows the time-frequency diagram of one TOPS burst. The definition of the TOPS timeline can be computed and optimized as suggested in [1], [2], resulting in the burst time $T_{\text {burst }}^{i}$ and the steering angle rate $k_{\varphi}^{i}$ (which results in $K_{\text {rot }}^{i}$ ) for each subswath $i$, so that the total cycle time is given by $T_{\text {cycle }}=\sum_{i} T_{\text {burst }}^{i}$. Concerning the signal properties, note in Fig. 2 that the total azimuth bandwidth spans several PRF intervals (the PRF is the gray area for a given time instant). As in the ScanSAR mode, the focused burst scene $T_{\text {scene }}$ is much larger than the raw data burst, requiring special care when performing the azimuth focusing. Note also the dependence of the Doppler centroid on the azimuth position of the target within a burst. This large Doppler variation introduces stringent requirements in the coregistration accuracy in order to avoid phase discontinuities at burst edges, which is the main topic to be covered in this paper. Section II states the problem and 


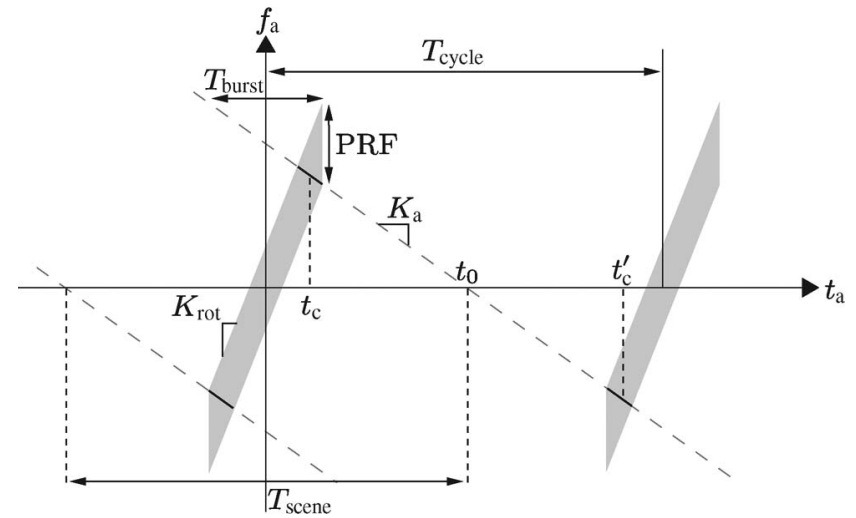

Fig. 2. Time-frequency diagram in the TOPS mode for two consecutive raw data bursts in the same subswath. The solid lines represent the targets observed within the main antenna beam (the gray area represents the instantaneous azimuth bandwidth). $t_{\mathrm{a}}$ is the azimuth time, $f_{\mathrm{a}}$ is the azimuth frequency, $T_{\text {cycle }}$ is the cycle time of the TOPS acquisition, $T_{\text {burst }}$ is the burst time, $T_{\text {scene }}$ is the duration of the observed scene for one burst, $t_{0}$ is the zero-Doppler time, $t_{\mathrm{c}}$ and $t_{\mathrm{c}}^{\prime}$ are the beam-center times for the target located at $t_{0}$ for the first and the second bursts, respectively, $K_{\mathrm{a}}$ is the Doppler rate of the target, and $K_{\text {rot }}$ is the Doppler centroid rate introduced by the antenna steering.

describes two approaches based on the spectral diversity (SD) or split-bandwidth technique [5], [7] in order to achieve the required coregistration accuracy. The use of stable point targets is also evaluated in Section II-F.

Section II-G addresses the effect of squint at burst edges. When focusing a burst, a wrong Doppler rate will introduce a shift in the impulse response function under the presence of a squint angle, which will turn into a burst misalignment. The effect in interferometric coregistration terms is also addressed.

Finally, Section III shows and validates the suggested approaches with real interferometric TOPS data acquired by the TerraSAR-X sensor over two different areas, including a performance assessment.

\section{ACCurate COREgistration OF TOPS PAirs}

\section{A. Problem Statement}

In a conventional squinted stripmap acquisition, a small constant coregistration error would induce a constant phase offset for the whole interferogram, hence not being critical. However, in the TOPS mode, a constant azimuth misregistration can cause the presence of an along-track linear phase ramp in the interferogram. This occurs because each TOPS burst is acquired with a varying Doppler centroid, and therefore every focused point has a different linear phase ramp in the azimuth direction. The resulting interferometric phase ramp leads then to phase jumps between bursts, which is obviously not desirable. The interferometric TOPS phase bias in the presence of azimuth misregistration is similar to the ScanSAR bias and is equal to [5]

$$
\phi_{\text {azerr }}(t)=2 \pi f_{\mathrm{DC}}(t) \Delta t
$$

where $t$ is the azimuth time, $f_{\mathrm{DC}}(t)$ is the azimuth-dependent Doppler centroid, and $\Delta t$ is the coregistration error in seconds. This corresponds to a linear interferometric phase bias along azimuth within each burst, since $f_{\mathrm{DC}}$ is azimuth dependent. As an example, for a TerraSAR-X acquisition with a Doppler centroid variation of $8.3 \mathrm{kHz}$, and assuming an image sampling of $0.001524 \mathrm{~s}$ (about $11 \mathrm{~m}$ at a ground velocity of $7100 \mathrm{~m} / \mathrm{s}$ ), a misregistration of 0.1 pixels introduces a ramp of approximately $2.5 \pi$ within the burst. Therefore, an overall azimuth coregistration accuracy better than 0.00065 of the pixel spacing is required for this configuration in order to achieve an error smaller than $3^{\circ}$.

\section{B. Interferometric Processing Strategy}

The proposed strategy in order to satisfy the requirements on the coregistration accuracy is the following. First, both master and slave images should be coregistered using a geometrical approach, i.e., by computing the offsets with the precise orbit information and an external digital elevation model (DEM) [8]. Note that with almost parallel orbits, as it is the case in TerraSAR-X, where the satellite is always steered to follow an orbital tube of $250 \mathrm{~m}$, the azimuth coregistration error becomes practically topography independent and hence turns into a constant value [8]. This means, that even taking an ellipsoidal model to compute the offsets this assumption would still hold. Nevertheless, in most cases, an external DEM of the area under study is available, hence being feasible to achieve very good relative coregistration accuracy, both in range and azimuth dimensions. Therefore, only a constant coregistration offset remains, imposed by the limited orbit accuracy. The TerraSAR-X science orbit has a 3-D $1 \sigma$ accuracy that can go down to $3 \mathrm{~cm}$ in periods of low solar activity [9], which would turn into an azimuth error of 0.003 pixels assuming an azimuth sampling of the final TOPS image of $11 \mathrm{~m}$. Therefore, once both master and slave images have been coregistered using the geometrical approach, it is still necessary to estimate a small constant coregistration error in azimuth. The same problem in the frame of interferometric ScanSAR processing was also investigated in [10], where the phase ramp given by (1) is estimated iteratively after precomputing and removing the topographic phase. The different solutions based on spectra diversity suggested in the present paper remove inherently the topographic component and offer the required accuracy in the estimation of the mutual shift.

\section{Spectral Diversity}

The SD technique [5] applied to an interferometric pair consists in splitting each of the two complex SAR images $s_{1}$ and $s_{2}$ in two images by filtering the upper and lower bands, resulting in four low-resolution images: $s_{1, u}, s_{1, l}, s_{2, u}, s_{2, l}$. The splitting can be done either in range or azimuth, being the latter, the one of interest in the present case. Then, two low-resolution interferograms are obtained by combining the images with the common spectra. A final interferogram between these two leads to a differential interferogram, which can be expressed as

$$
s_{\mathrm{sd}}=\left(s_{1, u} \cdot s_{2, u}^{*}\right) \cdot\left(s_{1, l} \cdot s_{2, l}^{*}\right)^{*} .
$$

The phase of this signal is given by [5]

$$
\phi_{\mathrm{sd}}=2 \pi \Delta f \Delta t
$$

where $\Delta f$ is the spectral separation of the two sublooks, $\Delta t$ is the coregistration error in seconds, and note that the topography component has been removed. This technique obtains a 
pixelwise estimation, which on the one hand is an advantage, but on the other hand imposes the condition of the coregistration error being smaller than the resolution cell of the used look. Also, since the differential interferogram is noisier than the normal interferogram, averaging is needed.

Concerning the performance of SD, consider first the Cramér-Rao bound in the estimation of the coregistration error, which is given by [7], [11]

$$
\sigma_{\mathrm{CR}}=\sqrt{\frac{3}{2 N}} \frac{\sqrt{1-\gamma^{2}}}{\pi \gamma}
$$

in units of resolution elements, where $N$ is the number of independent averaged samples, which in a practical case should consider the oversampling and the weighting window in both dimensions, and $\gamma$ is the interferometric coherence. As shown in [7] and briefly summarized in the following, SD can achieve this bound. The achievable accuracy in the estimation of the coregistration error with SD in terms of standard deviation of image samples is given by [7]

$$
\sigma_{\mathrm{sd}}=\frac{\sqrt{2} \sigma_{\mathrm{look}}}{2 \pi \Delta f} \frac{1}{\Delta t_{s}}
$$

where $\Delta t_{s}$ is the image sampling in seconds, and $\sigma_{\text {look }}$ is the interferometric phase standard deviation of one look given by

$$
\sigma_{\text {look }}=\sqrt{\frac{B}{b}} \sigma_{\text {int }}=\frac{1}{\sqrt{2 N}} \sqrt{\frac{B}{b}} \frac{\sqrt{1-\gamma^{2}}}{\gamma}
$$

where $\sigma_{\text {int }}$ is the phase standard deviation of the full resolution interferogram, $B$ is the processed bandwidth for a single target, and $b$ is the look bandwidth. As indicated in [7], when $b=$ $B / 3(\Delta f=B-b)$, the SD solution approaches the CramérRao bound in the estimation of the coregistration error given by (4). The conventional approach, which consists in interpolating the cross-correlation peak (coherent cross-correlation) between two single-look complex patches achieves also this bound.

\section{Enhanced Spectral Diversity (ESD)}

The enhanced SD (ESD) technique uses the burst overlap region in order to estimate more precisely the coregistration error. This technique was already suggested for ScanSAR [5] and is presented here in the frame of the TOPS mode. Fig. 3 shows the TOPS case with two consecutive focused bursts. The spectral separation in the overlap region, $\Delta f_{\text {ovl }}$, is much larger than the one achievable within the processed bandwidth, which is $\Delta f$. This spectral separation is responsible for the problematic interferometric azimuth phase ramp, but, at the same time, it gives more sensitivity when estimating the coregistration shift, as it will be demonstrated later in this section.

The spectral separation in the overlap region, $\Delta f_{\text {ovl }}$, can be computed from the geometry as shown in Fig. 2. The beamcenter time $t_{\mathrm{c}}$ is given by the intersection between the instantaneous frequency of the target located at $t_{0}$ and the Doppler centroid rate defined by the antenna steering, i.e.,

$$
K_{\text {rot }} t=K_{\mathrm{a}} \cdot\left(t-t_{0}\right)
$$

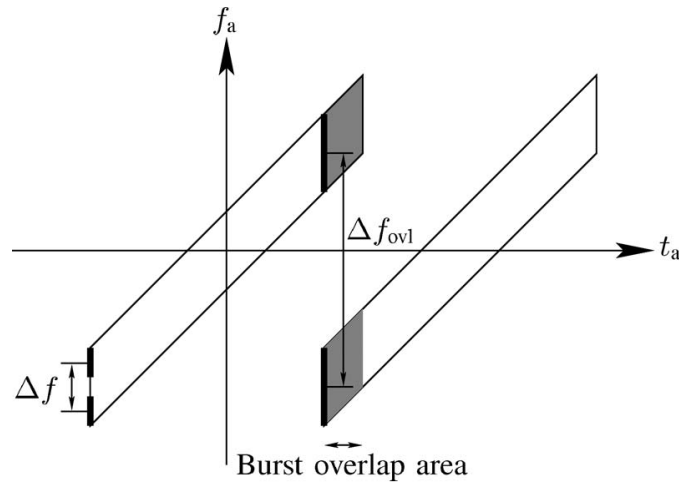

Fig. 3. Time-frequency diagram of two consecutive focused bursts sketching the enhanced spectral diversity approach. The targets located in the overlap region (shown in gray) have a large spectral separation $\Delta f_{\text {ovl }}$, which gives a higher sensitivity to the azimuth coregistration offset. The conventional SD approach uses only $\Delta f$, which is limited by the processed bandwidth.

where $t$ is the azimuth time, $t_{0}$ is the zero-Doppler position of the target, $K_{\mathrm{a}}$ is the Doppler rate of the target, and $K_{\text {rot }}$ is the Doppler centroid rate introduced by the antenna steering. The last two are given by

$$
\begin{aligned}
K_{\mathrm{a}} & =-\frac{2 v_{\mathrm{eff}}^{2}}{\lambda r_{0}} \\
K_{\mathrm{rot}} & =-\frac{2 v_{\mathrm{eff}}^{2}}{\lambda r_{\mathrm{rot}}} \simeq \frac{2 v_{\mathrm{s}}}{\lambda} k_{\varphi}
\end{aligned}
$$

where $r_{\text {rot }}$ is the distance to the virtual rotation center of the acquisition, which is negative as it is located behind the sensor in the TOPS case [12]. Solving (7) for $t$ gives

$$
t_{\mathrm{c}}=\frac{K_{\mathrm{a}}}{K_{\mathrm{a}}-K_{\mathrm{rot}}} t_{0} .
$$

Similarly, the same target will be observed $T_{\text {cycle }}$ seconds later in the following burst, so that:

$$
t_{\mathrm{c}}^{\prime}=\frac{K_{\mathrm{a}} t_{0}-K_{\text {rot }} T_{\text {cycle }}}{K_{\mathrm{a}}-K_{\text {rot }}}=t_{\mathrm{c}}-\frac{K_{\mathrm{a}}}{K_{\mathrm{a}}-K_{\text {rot }}} T_{\text {cycle }} .
$$

The desired spectral separation is then given by

$$
\Delta f_{\text {ovl }}=\left|K_{\mathrm{a}} \cdot\left(t_{\mathrm{c}}^{\prime}-t_{\mathrm{c}}\right)\right|=\left|\frac{K_{\mathrm{a}} K_{\text {rot }}}{K_{\mathrm{a}}-K_{\text {rot }}} T_{\text {cycle }}\right| .
$$

Therefore, taking (5) and (12), the accuracy in the estimation of the coregistration error with ESD in terms of image samples is given by

$$
\sigma_{\text {ovl }}=\frac{\sqrt{2} \sigma_{\text {int }}}{2 \pi \Delta f_{\text {ovl }}} \frac{1}{\Delta t_{s}}=\frac{1}{2 \pi \Delta f_{\text {ovl }}} \frac{1}{\sqrt{N}} \frac{\sqrt{1-\gamma^{2}}}{\gamma} \frac{1}{\Delta t_{s}}
$$

where $\sigma_{\text {int }}$ is used instead of $\sigma_{\text {look }}$, since the whole bandwidth $B$ is being used in this case. Note also that $\Delta f_{\text {ovl }}$ is range dependent, but this dependency can be neglected for typical spaceborne configurations.

An interesting point to note is that the coregistration accuracy requirement and the achievable accuracy compensate each other, i.e., the larger the maximum steering angles, the larger also the sensitivity to the coregistration error when using the 

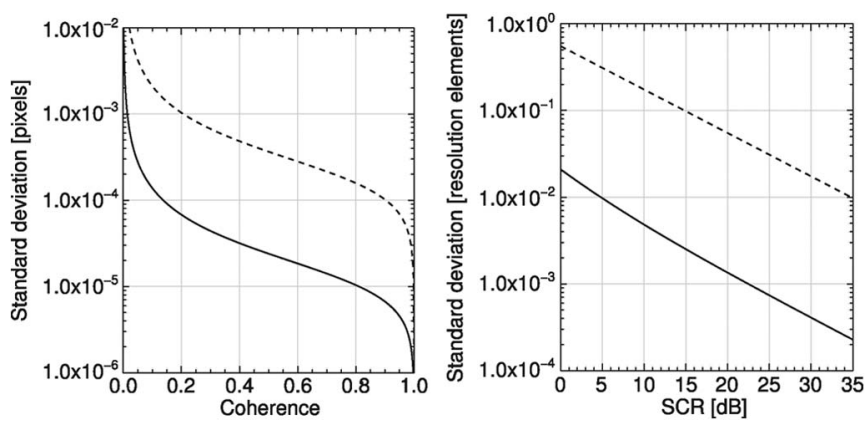

Fig. 4. (Left) Accuracy in the estimation of the constant azimuth coregistration shift as a function of the coherence (dashed) when using SD within one burst and (solid) when using the overlap region between consecutive bursts, i.e., ESD. (Right) Accuracy in the estimation of the coregistration shift in units of resolution elements for a single point scatterer as a function of the SCR, with the (dashed) conventional approach and (solid) ESD. The system and processing parameters of Table I have been used. A logarithmic scale has been used for the vertical axis.

ESD approach. This can be shown by taking (1), so that the accuracy of the interferometric phase bias due to a coregistration error is given by

$$
\begin{aligned}
\sigma_{\phi_{\mathrm{azerr}}} & =2 \pi f_{\mathrm{DC}} \sigma_{\mathrm{ovl}} \Delta t_{s}=\sqrt{2} \sigma_{\mathrm{int}} \frac{f_{\mathrm{DC}}}{\Delta f_{\mathrm{ovl}}} \\
& =\sqrt{2} \sigma_{\mathrm{int}} \frac{T_{B}}{2 T_{\text {cycle }}}\left(1-\frac{K_{\mathrm{rot}}}{K_{\mathrm{a}}}\right)
\end{aligned}
$$

where $T_{B}$ is the burst time, and $f_{\mathrm{DC}}=K_{\mathrm{rot}} T_{B} / 2$ is the Doppler centroid at the burst edge. In order to have a TOPS timeline without gaps between bursts and assuming $T_{\text {cycle }}=$ $N_{s} T_{B}$, being $N_{s}$ the number of subswaths, the following condition must be satisfied [1]:

$$
K_{\mathrm{rot}} \geq\left|K_{\mathrm{a}}\right| \cdot\left(1-N_{s}\right)
$$

By taking the lower bound, (15) turns into

$$
\sigma_{\phi_{\text {azerr }}}=\frac{\sigma_{\text {int }}}{\sqrt{2}}
$$

i.e., the final accuracy of the phase bias due to a coregistration error is independent of the Doppler centroid and just dependent on the phase quality of the interferogram and the number of looks used to average the SD phase. For this particular case, $\Delta f_{\text {ovl }}=2 f_{\mathrm{DC}}$, but the overlap region is theoretically just one pixel. In practice, a certain amount of overlap is desired to be able to use several pixels to achieve a better performance. Therefore, $\Delta f_{\text {ovl }}$ will be always smaller than $2 f_{\mathrm{DC}}$, having then a tradeoff between the amount of overlap and the spectral separation, being the latter the one that has a larger impact in the performance. In any case, increasing the amount of overlap does not decrease significantly the spectral separation.

\section{E. Performance Evaluation for TerraSAR-X}

Fig. 4 (left) shows the expected performance in the estimation of the coregistration shift using SD and ESD for a given TerraSAR-X TOPS interferometric pair, whose image parameters are given in Table I (first subswath). The performance is shown for one single burst in the SD case, and for one single
TABLE I

Main System and Processing Parameters of the TerraSAR-X TOPS ACQUiSITION OVER THE ATACAMA DESERT

\begin{tabular}{lc}
\hline Wavelength & $3.106 \mathrm{~cm}$ \\
Sub-swaths & 4 \\
Bursts / sub-swath & 14 \\
PRFs & $4380 / 4044 / 3728 / 3423 \mathrm{~Hz}$ \\
Sampling frequency & $109.88 \mathrm{MHz}$ \\
Chirp bandwidth & $100 \mathrm{MHz}$ \\
Effective velocity & $7394.27 \mathrm{~m} / \mathrm{s}$ \\
Burst durations & $0.32 / 0.35 / 0.40 / 0.44 \mathrm{~s}$ \\
Cycle time & $1.528 \mathrm{~s}$ \\
Range sampling & $1.36 \mathrm{~m}$ \\
Azimuth sampling & $0.001524 \mathrm{~s}(\sim 10.8 \mathrm{~m})$ \\
Mid range & $633 / 647 / 663 / 680 \mathrm{~km}$ \\
Rotation range & $101 /-117 /-155 /-176 \mathrm{~km}$ \\
Samples per burst & $16 / 17 / 19 / 20 \times 10^{6}$ \\
Samples in overlap region & $1.8 / 1.5 / 3.0 / 3.1 \times 10^{6}$ \\
$\Delta f_{\text {ovl }}$ & $7.3 / 7.0 / 6.5 / 6.2 \mathrm{kHz}$ \\
Scene size & $150 \mathrm{~km} \times 100 \mathrm{~km}($ azimuth $\times$ range $)$ \\
\hline
\end{tabular}

overlap region in the ESD case. Note that all available pixels have been averaged, i.e., $16 \times 10^{6}$ for $\mathrm{SD}$ and $1.8 \times 10^{6}$ for ESD, since a constant offset for the whole burst is assumed. As expected, the accuracy obtained using the overlap burst region is better than using one burst, since the final accuracy improves inversely proportional to the spectral separation of the looks, while only inversely proportional to the square root of the number of averaged samples. Nevertheless, the performance of both approaches is sufficient to estimate the shift with the desired accuracy (better than 0.00065 pixels in the TerraSAR$\mathrm{X}$ case), even for very low coherence values. In addition to the better performance of the second approach, it has also the advantage that the looks are readily available, i.e., only the overlap region of the successive burst interferograms needs to be combined to generate the SD phase. This saves computational load compared to the first approach, where the looks need to be generated explicitly. On the other hand, this approach has an important drawback: Due to the larger sensitivity, the SD phase might be wrapped. The maximum coregistration error in pixels that can be measured in ESD without aliasing is

$$
\Delta p_{\max }= \pm \frac{1}{2 \Delta f_{\text {ovl }} \cdot \Delta t_{s}} .
$$

Using (12) and the parameters of Table I, for this particular case, this value is equal to \pm 0.045 pixels on the first subswath. Therefore, it must be ensured that the residual coregistration error is smaller than this limit, since otherwise a wrapped (wrong) value will be estimated. Note, however, that thanks to the TerraSAR-X science orbit accuracy, the residual coregistration error to be estimated will be in most cases smaller than this limit ( $3 \mathrm{~cm}$ absolute azimuth position accuracy correspond to 0.003 TerraSAR-X azimuth TOPS pixels). Alternatively, a previous estimation of the residual error with cross-correlation or conventional SD can be used to unwrap the ESD estimation, if required.

A further topic to address is the variation of the azimuth coregistration error during the data take. It might be assumed that the orbit accuracy remains constant throughout the data take in the sense of a constant azimuth position offset. Then, the 
residual coregistration error will also be azimuth invariant. In this case, the individual burst (SD) or overlap region (ESD) estimation results can be averaged, provided the timing information of the sensor is accurately considered by the processor during burst alignment. However, this assumption might not hold for long data takes. The TerraSAR-X science orbit is computed using the most accurate geophysical models, including considerations for nongravitational perturbations such as solar radiation pressure, atmospheric drag, or orbit maintenance manoeuvers. Furthermore, unmodeled errors are estimated every $10 \mathrm{~min}$ assuming a set of empirical 3-D accelerations [9]. Therefore, it is plausible that the orbit position bias might vary slightly when coming close to these $10 \mathrm{~min}$. In any case, note that the required azimuth coregistration accuracy can be reached using ESD with just one single overlap region. Hence, the residual error might be estimated and corrected independently for every burst, e.g., assuming a low-order polynomial fit of the individual estimates, such that any variations in the orbit accuracy would be properly taken into account.

\section{F. Point Scatterers}

As also shown in [7], the accuracy in the estimation of the coregistration error using point scatterers in incoherent clutter is given by

$$
\sigma_{\text {point }}=\frac{\sqrt{3}}{\pi} \frac{1}{\sqrt{\mathrm{SCR}}}
$$

in units of resolution elements, where SCR is the signal-toclutter ratio. This curve is plotted with dashed style in Fig. 4 (right), and obviously the performance does not suffice in order to achieve the requirements with a single point with a realistic SCR, particularly when considering the relatively low compression gain of TOPS data. Therefore, several correlation results need to be averaged. As an example, assuming point scatterers with a SCR of $10 \mathrm{~dB}$, one would need to average about $70 \times 10^{3}$ of such points to achieve an accuracy of 0.00065 resolution elements. This approach might be viable only in an urban scenario.

Instead, one can also use ESD with point scatterers laying in the burst overlap region. The interferometric phase standard deviation of a point target with a sufficiently large SCR $(>4 \mathrm{~dB})$ is given by [13]

$$
\sigma_{\mathrm{int}, \mathrm{point}} \approx \frac{1}{\sqrt{\mathrm{SCR}}} \sqrt{1+\frac{5}{12 \cdot \mathrm{SCR}}}
$$

so that by taking again (13), the resulting standard deviation in the measurement of the coregistration error using point targets and ESD is

$$
\sigma_{\text {ovl,point }}=\frac{\sqrt{2} \sigma_{\text {int,point }}}{2 \pi \Delta f_{\text {ovl }}} \cdot B
$$

in units of resolution elements. Fig. 4 (right) plots this curve with solid style against different SCR values using a spectral separation of $\Delta f_{\text {ovl }}=7.3 \mathrm{kHz}$, as given in Table I for the first subswath. In this case, assuming again point targets with a SCR of $10 \mathrm{~dB}$, one would need just about 40 of such targets at the overlap region in order to achieve an accuracy of 0.00065 pixels, which turns out to be much more feasible than in the previous case.

\section{G. SAR Focusing Discussion}

A further point to analyze is the impact of the topography in the focusing of a TOPS burst. The effective velocity in the spaceborne case depends slightly on the altitude of each target due to the curved orbit. In other words, targets at the same zero-Doppler range distance but with different altitudes have slightly different phase histories. When processing with efficient FFT-based processors, the same effective velocity must be used for a large block of data, implying a potential mismatch between the target's Doppler rate and the one used during azimuth compression. The difference is usually small and has a negligible impact in terms of focusing quality for conventional SAR systems. However, the impulse response will be shifted under the presence of squint. Effectively, a wrong effective velocity introduces the following shift in the impulse response [14]:

$$
\delta t=f_{\mathrm{DC}} \cdot\left(\frac{1}{K_{a}}-\frac{1}{\tilde{K}_{a}}\right)
$$

where the tilde indicates the Doppler rate with the mismatch in the effective velocity. Note that the induced shift is wavelength independent, since it cancels out with the quotient between the Doppler centroid and the Doppler rate. In the particular TOPS case, the points in the overlap region have a different Doppler centroid, which for a mean zero-squinted acquisition has the same absolute value but an opposite sign. Therefore, in the presence of a mismatch in the Doppler rate, the targets will be separated by

$$
\delta t=\Delta f_{\mathrm{ovl}} \cdot\left(\frac{1}{K_{a}}-\frac{1}{\tilde{K}_{a}}\right)
$$

seconds. This coregistration error corresponds to a subsequent burst misalignment, which is space variant due to the topography dependence. Fig. 5 shows a plot of the introduced shift in pixels as a function of the topography and the squint separation for the system parameters of Table I (first subswath). As observed, this error can reach one tenth of a pixel for large topographic differences, but still having a minor impact in the focusing quality.

The above development assumes a perfect match of the reference function for a reference height. This implies that the hyperbolic approximation, which assumes a straight orbit, and which is used with current FFT-based processors like [12], still applies, as it is the case when dealing with small observation times and small squint angles. For larger observation times and/or squint angles, a numerical approach like the one suggested in [15] could be used in order to achieve the best fit of the focusing kernel in the L2 norm sense. Nevertheless, the potential Doppler rate mismatch due to the topography would still be present. If needed, a block-based postprocessing approach could accommodate the topography and remove the aforementioned shift. Similarly, a time-domain 


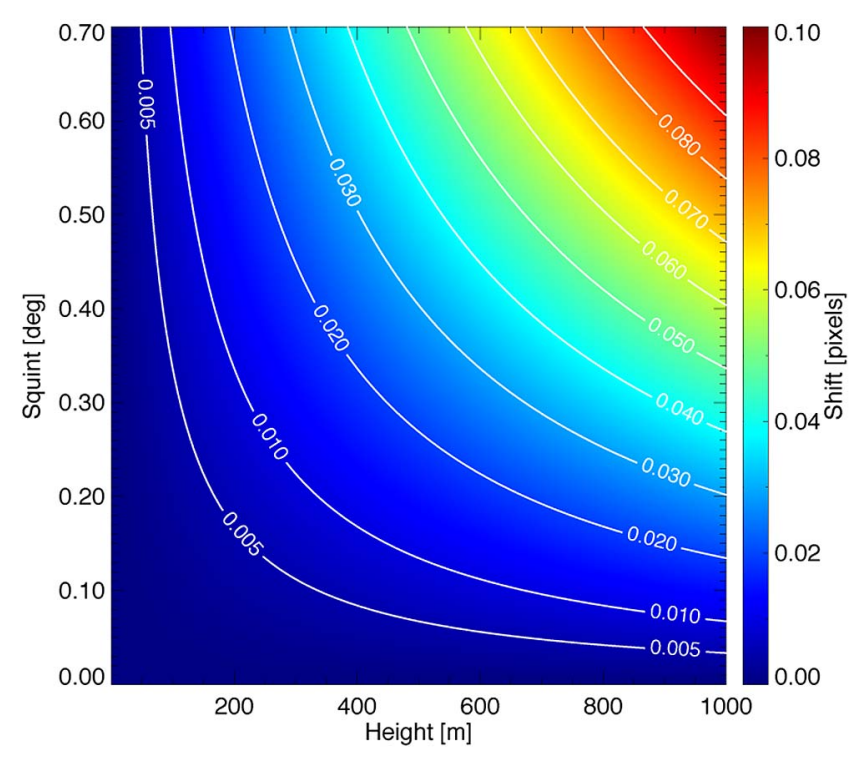

Fig. 5. Introduced shift in the impulse response function in pixel units as a function of the squint angle and the topographic height difference with respect to the reference one. The system parameters of Table I have been used for the numerical evaluation.

processor might accommodate the topography in a pixelwise fashion, with the corresponding tradeoff between accuracy and efficiency [16].

The shift due to the Doppler rate mismatch might seem to have a big impact in the interferometric performance, but note that this will depend on the relative difference between the errors in the effective velocities for the master and slave images. If the relative difference is zero, then the points will be shifted by exactly the same amount given in (23), hence having no impact in terms of interferometric coregistration. However, this does not need to be always the case. Under large baselines or nonparallel orbits, the mean effective velocity along azimuth for a given block might change due to the different projection of the topography in the line of sight. In such cases, the relative difference between effective velocity errors is nonzero, and thus a residual coregistration error between two consecutive bursts will be introduced, which will be space variant and will bias the estimation of the constant coregistration offset. A numerical evaluation should be performed in every case in order to quantify this error, provided an external DEM is available. If needed, the computed residual coregistration error map can be used to further coregister the slave image and remove the space-variant component. For the data presented in this paper, the numerical evaluation yielded insignificant residual errors, given the TerraSAR-X orbit is controlled within an orbital tube of $250 \mathrm{~m}$.

\section{EXPERIMENTAL RESUlts}

This section shows interferometric results with real TOPS data acquired by TerraSAR-X. Two data sets are used: A low coherence data set over Mexico City, which also includes subsidence effects, and a good coherence data set over the Atacama desert, Chile, which is also used in Section III-C for the performance validation in the estimation of the coregistration shift. The data have been processed using the experimental
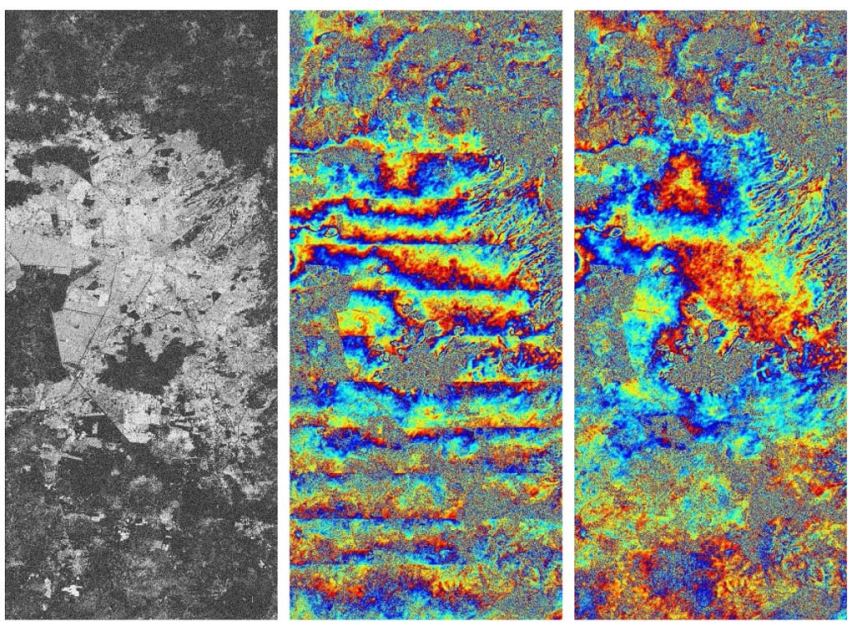

Fig. 6. (Left) TOPS interferometric coherence for one subswath and (middle and right) residual phases, i.e., after subtracting the topographic fringes. (Middle) With an artificial coregistration error of 0.05 samples. The phase jumps at burst edges are about $145^{\circ}$, which in this case would turn into a DEM discontinuity of $45 \mathrm{~m}$ between bursts, or similarly to a jump of $6 \mathrm{~mm}$ in the differential InSAR case. (right) After estimating the error using ESD and correcting the shift, where the jumps are no longer visible. Range is horizontal, and azimuth is vertical.

TanDEM-X Interferometric Processor (TAXI) [17], which uses the baseband azimuth scaling approach [12] for the dedicated focusing of the TOPS data.

\section{A. Mexico City Example}

Fig. 6 shows the effect of an uncorrected azimuth coregistration error in a TOPS interferogram with a data take acquired over Mexico City with a time baseline of 22 days. The synthetic phase computed using a SRTM DEM has been subtracted from the interferogram. Here, only the third subswath is shown, where the number of bursts is 13 and its size is $30 \mathrm{~km} \times$ $100 \mathrm{~km}$. An error of 0.05 samples was induced on purpose to clearly visualize the phase jumps. Such an error introduces a phase jump of $145^{\circ}$ between bursts, which would result in a DEM discontinuity of $45 \mathrm{~m}$ between bursts for the given baseline, or similarly in a jump of $6 \mathrm{~mm}$ in the case of differential interferometry. After the correction, no phase jumps between bursts are visible anymore.

Fig. 7 shows the results for the complete data take over Mexico City after the accurate coregistration using in this case a pair with 4 months separation. The scene dimensions are $100 \mathrm{~km} \times$ $100 \mathrm{~km}$ with 13 bursts per subswath. Despite the large time baseline and low coherence, the residual coregistration error could be properly estimated and corrected, as no phase jumps are visible. On the other hand, since the topographic phase was subtracted, most of the fringes in the main part of the city area correspond to real deformation due to ground water extraction [18], [19]. Fig. 8 shows the measured subsidence. The atmospheric phase screen (APS) was not removed, and there might also be residual topographic errors. Nevertheless, note that assuming a maximum APS of $1 \mathrm{~cm}$, the maximum observed deformation after 4 months is about one order of magnitude larger than these residual errors. 

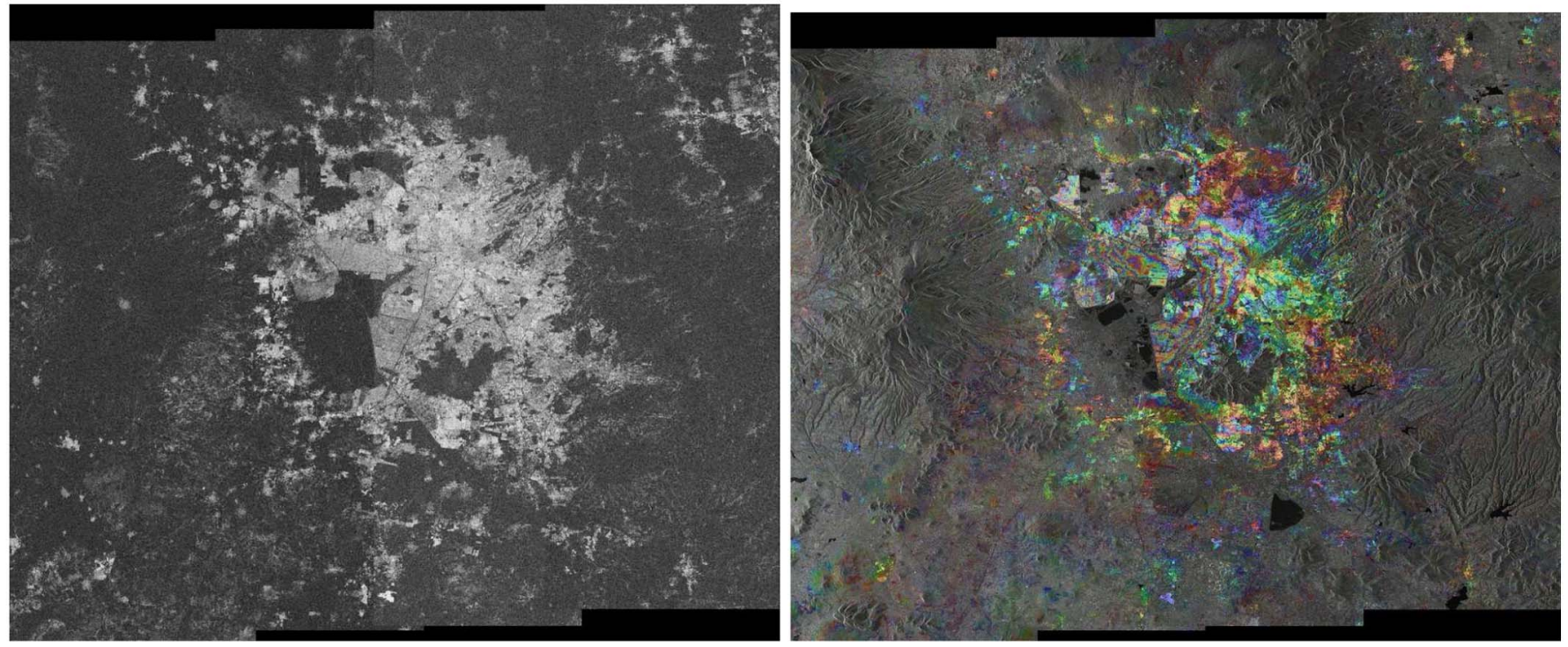

Fig. 7. Interferometric TOPS data take over Mexico City acquired by TerraSAR-X showing the (left) coherence and the (right) residual interferometric phase with overlaid reflectivity. Range is horizontal, and azimuth is vertical.

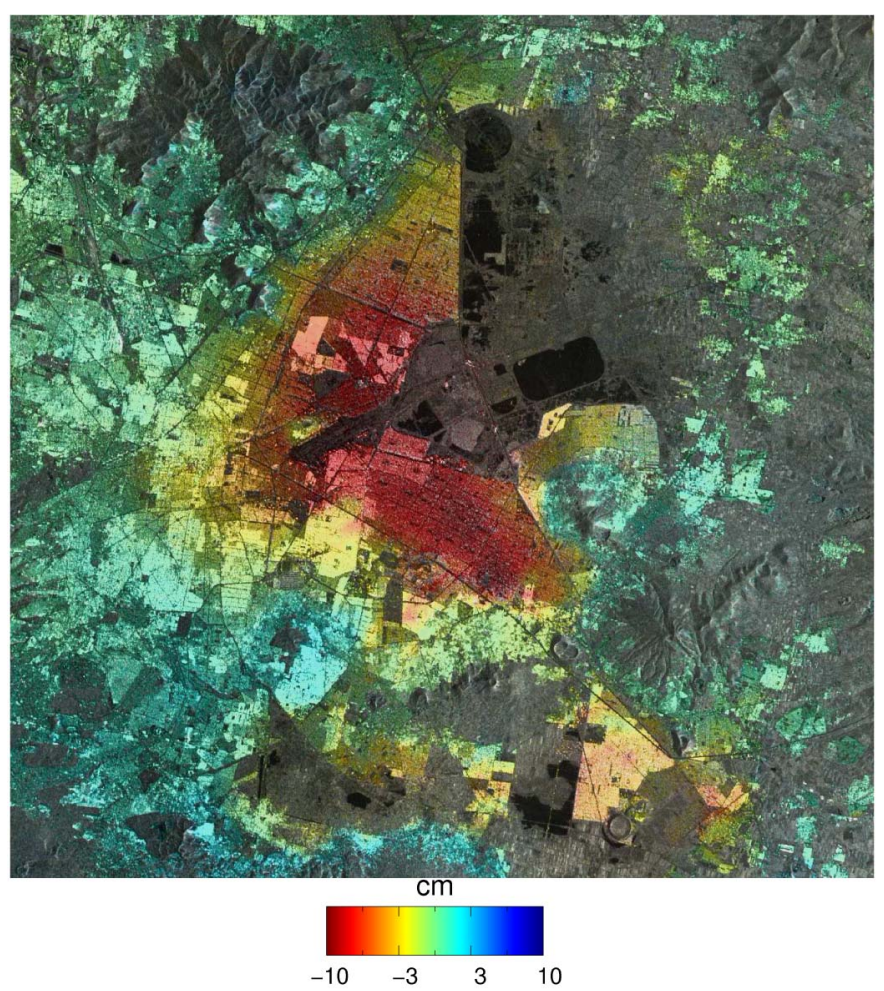

Fig. 8. Geocoded image of the measured deformation over Mexico City with two TOPS TerraSAR-X images acquired with 4 months difference. A subsidence greater than $10 \mathrm{~cm}$ can be observed in some city areas.

\section{B. Atacama Desert Example}

Fig. 9 shows the azimuth phase gradient of the first three overlap regions of the first subswath corresponding to an interferometric pair over the Atacama desert, at the Chile-Argentina border. The interferometric pair was acquired with 11 days difference, but due to the extreme dry conditions and lack of vegetation, the coherence is very high (mean of 0.91). In this case, no error was induced on purpose, so that the small phase

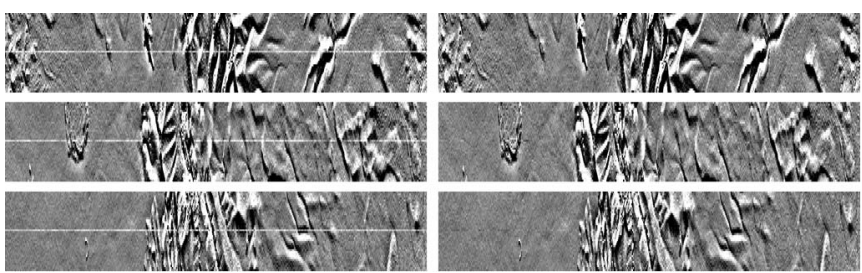

Fig. 9. Azimuth phase gradient of the TOPS residual phases of the first three overlap regions of the Atacama desert data take. The area shown covers the whole subswath range extension, but only 40 pixels around the overlap region. The scaling goes from $-30^{\circ}$ (black) to $30^{\circ}$ (white). (left) With a coregistration error of 0.00438 samples (estimated a posteriori), where the phase jumps between bursts are visible as white lines. The white lines have a value of about $18^{\circ}$, which matches the estimated coregistration error. With the given baseline, this value would correspond to a systematic jump of $3 \mathrm{~m}$ between bursts in the final DEM, or a jump of $0.8 \mathrm{~mm}$ in the differential InSAR case. (right) After the estimation and shift of the residual coregistration error. Range is horizontal, and azimuth is vertical.
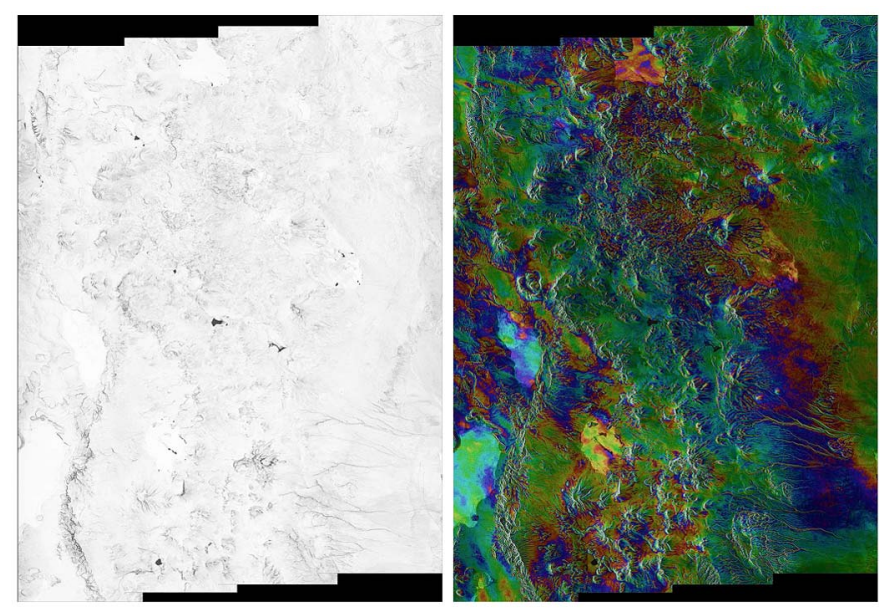

Fig. 10. Interferometric TOPS data take over the Atacama desert at the Chile-Argentina border acquired by TerraSAR-X showing the (left) coherence and (right) residual interferometric phase with overlaid reflectivity. Range is horizontal, and azimuth is vertical. 

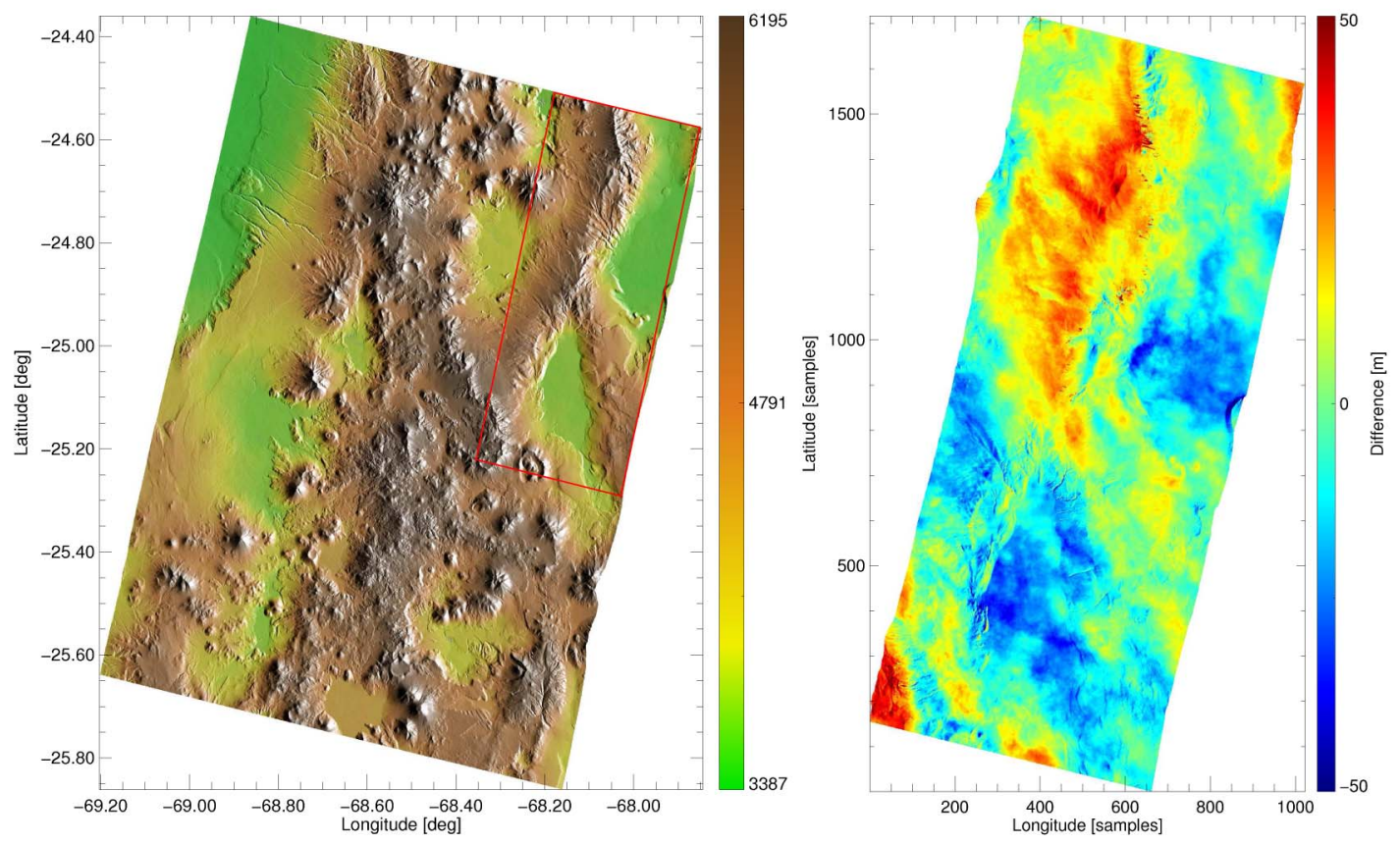

Fig. 11. (Left) DEM generated with two TOPS repeat-pass acquisitions. (Right) Difference between TOPS and stripmap DEMs for the red rectangle shown in the figure on the left. Most of the errors are attributed to atmospheric artifacts.

jumps one can observe are due to the real error resulting from the orbit accuracy. The estimated offset in this case is 0.00438 samples, which corresponds to $4.7 \mathrm{~cm}$ and agrees with the expected orbit accuracy. The phase jump between bursts has a value of about $18^{\circ}$, which with the given baseline would correspond to a discontinuity of $3 \mathrm{~m}$ between bursts in the final DEM, or a jump of $0.8 \mathrm{~mm}$ in the differential InSAR case. After the correction the phase jumps have disappeared.

Fig. 10 shows the whole TOPS data take over the Atacama desert, including the coherence and the residual phase with the reflectivity image overlaid. This residual phase contains mainly DEM errors and atmospheric artifacts. The scene dimensions are $150 \mathrm{~km} \times 100 \mathrm{~km}$ with 14 bursts per subswath. The generated DEM is shown in Fig. 11, as well as the difference with another one obtained from repeat-pass TerraSAR-X stripmap data. The difference between both DEMs has a standard deviation of $15 \mathrm{~m}$, and no specific artifacts at burst edges can be observed. The perpendicular baselines are $115 \mathrm{~m}$ (TOPS) and $138 \mathrm{~m}$ (stripmap), yielding a height of ambiguity of $51 \mathrm{~m}$ and $42 \mathrm{~m}$, respectively. With these numbers, an atmospheric error of $5 \mathrm{~mm}$ would already correspond to $30 \mathrm{~m}$ in the DEM difference, which is considered to be the main source of error, as shown on the second plot of Fig. 11.

\section{Performance Assessment With Real Data}

The same TOPS data take over the Atacama desert is used to evaluate the expected performance as derived in Section II. The mean coherence of this data take is 0.91 , and the estimated coregistration error has a value of 0.00438 samples, both with conventional SD and ESD. The difference between both results is just of $3 \times 10^{-5}$ pixels, which is already below the accuracy of SD. For SD, the coregistration error has been estimated with
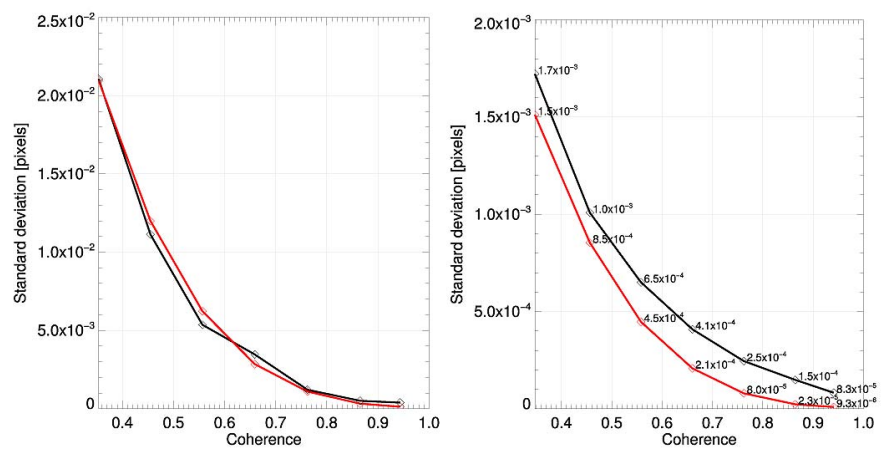

Fig. 12. Standard deviation in the estimation of the coregistration error with (left) conventional SD and (right) ESD. Estimated (black) and theoretical (red) standard deviation of the measured coregistration error.

each of the 56 available bursts in order to compute the standard deviation of the estimations. Similarly, the estimations over the 52 overlap regions have been used to compute the accuracy of ESD. Furthermore, the measurement has been performed using different coherence ranges. The black solid line in Fig. 12 shows the resulting standard deviation for the corresponding coherences, while the red solid line shows the expected performance as derived from (5) and (13), respectively. Note that the theoretical curves consider the oversampling and the weighting window of the data, since these have a direct impact on the effective number of independent averaged samples. The results for the SD case are very similar to the theoretical ones. In the ESD case, the accuracy is in the worst case almost one order of magnitude worse than expected. However, one has to consider that we are dealing with real data, and more realizations would be necessary to properly quantify the performance. Further, the whole processing must be accurately performed. In particular, the burst alignment during the focusing step is critical, since 
the presence of even very small different offsets from one burst to the next would introduce a bias in the estimated values. In any case, note that the achieved performance of the ESD approach is satisfactory and approximately one order of magnitude better than that of the conventional SD or crosscorrelation approaches.

\section{CONCLUSion}

This paper has analyzed the requirements in terms of interferometric coregistration accuracy for the TOPS mode. It has been shown that even a small constant azimuth coregistration shift can introduce severe azimuth phase ramps in each burst. These occur due to the azimuth-varying Doppler centroid of the TOPS signal similar as in the ScanSAR case, although in the former, the variation is much larger and mainly due to the steering of the antenna. In the case of a small orbital tube, as is the case with TerraSAR-X, the coregistration error in azimuth is almost constant for the whole image and insensitive to the topography [8], which eases the estimation procedure and allows one to assume that only a constant offset for the whole image is present. Note that in most practical cases, an external DEM will be used, e.g., SRTM, which further supports this assumption. Hence, the coregistration offset is mainly due to the error of the orbit product $(3-20 \mathrm{~cm}$ in the science orbit product of TerraSAR-X [9]).

Two approaches to estimate this residual azimuth coregistration offset have been expounded, and their performance evaluated. The first approach is the conventional cross-correlation technique, or alternatively, the SD technique, which can achieve satisfactory results since many independent samples can be averaged. However, the recommended technique suggested in this paper is the enhanced SD technique (ESD). This approach uses the information of the pixels within the overlap region between bursts, which contains pixels that have been observed with different squint angles, hence having a higher sensitivity to the coregistration error. Even with a reduced overlap region, better performances than with the conventional SD case are obtained. Finally, the exploitation of point scatterers with ESD seems also feasible, while the conventional SD or cross-correlation requires a much larger number of such points in order to achieve the required accuracy.

The effect of squint in focusing terms has been also analyzed. A wrong Doppler rate will shift the impulse response function for targets located at burst edges, hence introducing a burst misalignment. This shift can also be problematic in interferometric terms for large baselines or nonparallel orbits. A numerical evaluation using an external DEM should be performed in every case in order to quantify the magnitude of the error and, if needed, correct it.

Two different interferometric pairs acquired by TerraSAR-X, one over Mexico City and one over the Atacama desert at the Chile-Argentina border, have been used to validate the proposed approaches. Results have been shown concerning interferometric burst alignment accuracy, TOPS-stripmap DEM comparison, and first TOPS DInSAR results. Most important, real data have been used to validate the theoretical accuracy prediction.

\section{ACKNOWLEDGMENT}

The authors would like to thank Dr. F. De Zan and M. Rodríguez-Cassolà for the useful discussions on some topics covered in the paper. The authors would also like to acknowledge the anonymous reviewers for their valuable comments and suggestions, which helped to improve the quality of the paper.

\section{REFERENCES}

[1] F. De Zan and A. Monti Guarnieri, "TOPSAR: Terrain observation by progressive scans," IEEE Trans. Geosci. Remote Sens., vol. 44, no. 9, pp. 2352-2360, Sep. 2006.

[2] A. Meta, J. Mittermayer, P. Prats, R. Scheiber, and U. Steinbrecher, "TOPS imaging with TerraSAR-X: Mode design and performance analysis," IEEE Trans. Geosci. Remote Sens., vol. 48, no. 2, pp. 759-769, Feb. 2010.

[3] E. Attema, P. Bargellini, P. Edwards, G. Levrini, S. Lokas, L. Moeller, B. Rosich-Tell, P. Secchi, R. Torres, M. Davidson, and P. Snoeij, "Sentinel-1: The radar mission for GMES operational land and sea services," European Space Agency, ESA Bulletin 131, 2007.

[4] M. Bara, R. Scheiber, A. Broquetas, and A. Moreira, "Interferometric SAR signal analysis in the presence of squint," IEEE Trans. Geosci. Remote Sens., vol. 38, no. 5, pp. 2164-2178, Sep. 2000.

[5] R. Scheiber and A. Moreira, "Coregistration of interferometric SAR images using spectral diversity," IEEE Trans. Geosci. Remote Sens., vol. 38, no. 5, pp. 2179-2191, Sep. 2000.

[6] G. Fornaro, E. Sansosti, R. Lanari, and M. Tesauro, "Role of processing geometry in SAR raw data focusing," IEEE Trans. Aerosp. Electron. Syst., vol. 38, no. 2, pp. 441-454, Apr. 2002.

[7] R. Bamler and M. Eineder, "Accuracy of differential shift estimation by correlation and split-bandwidth interferometry for wideband and Delta-k SAR systems," IEEE Geosci. Remote Sens. Lett., vol. 2, no. 2, pp. 151155, Apr. 2005.

[8] E. Sansosti, P. Berardino, M. Manunta, F. Serafino, and G. Fornaro, "Geometrical SAR image registration," IEEE Trans. Geosci. Remote Sens., vol. 44, no. 10, pp. 2861-2870, Oct. 2006.

[9] Y. T. Yoon, M. Eineder, N. Yague-Martinez, and O. Montenbruck, "TerraSAR-X precise trajectory estimation and quality assessment," IEEE Trans. Geosci. Remote Sens., vol. 47, no. 6, pp. 1859-1868, Jun. 2009.

[10] B. Ortiz and H. Zebker, "ScanSAR-to-Stripmap mode interferometry processing using ENVISAT/ASAR data," IEEE Trans. Geosci. Remote Sens., vol. 45, no. 11, pp. 3468-3480, Nov. 2007.

[11] F. De Zan, "Coherent shift estimation for stacks of SAR images," IEEE Geosci. Remote Sens. Lett., vol. 8, no. 6, pp. 1095-1099, Nov. 2011.

[12] P. Prats, R. Scheiber, J. Mittermayer, A. Meta, and A. Moreira, "Processing of sliding spotlight and TOPS SAR data using baseband azimuth scaling," IEEE Trans. Geosci. Remote Sens., vol. 48, no. 2, pp. 770-780, Feb. 2010.

[13] W. Ye, T. S. Yeo, and Z. Bao, "Weighted least-squares estimation of phase errors of SAR/ISAR autofocus," IEEE Trans. Geosci. Remote Sens., vol. 37, no. 5, pp. 2487-2494, Sep. 1999.

[14] J. C. Curlander and R. N. McDonough, Synthetic Aperture Radar: Systems and Signal Processing. New York: Wiley, 1991.

[15] D. D'Aria and A. Monti Guarnieri, "High-resolution spaceborne SAR focusing by SVD-stolt," IEEE Geosci. Remote Sens. Lett., vol. 4, no. 4, pp. 639-643, Oct. 2007.

[16] L. M. H. Ulander, H. Hellsten, and G. Stenström, "Synthetic aperture radar processing using fast factorized back-projection," IEEE Trans. Aerosp. Electron. Syst., vol. 39, no. 3, pp. 760-776, Jul. 2003.

[17] P. Prats, M. Rodriguez-Cassola, L. Marotti, M. Naninni, S. Wollstadt, D. Schulze, N. Tous-Ramon, M. Younis, G. Krieger, and A. Reigber, "TAXI: A versatile processing chain for experimental TanDEM-X product evaluation," in Proc. IEEE IGARSS, Honolulu, HI, Jul. 25-30, 2010, pp. 4059-4062.

[18] T. Strozzi, U. Wegmüller, C. L. Werner, A. Wiesmann, and V. Spreckels, "JERS SAR interferometry for land subsidence monitoring," IEEE Trans. Geosci. Remote Sens., vol. 41, no. 7, pp. 1702-1708, Jul. 2003.

[19] L. Marotti, P. Prats, R. Scheiber, S. Wollstadt, and A. Reigber, "TOPS differential SAR interferometry with TerraSAR-X," in Proc. FRINGE, Frascati, Italy, Sep. 2011, p. 96. 


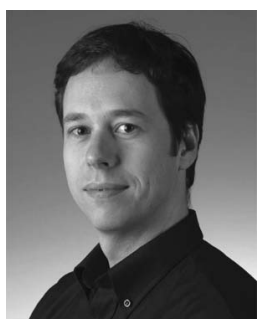

Pau Prats-Iraola (S'03-M'06) was born in Madrid, Spain, in 1977. He received the Ingeniero degree in telecommunication engineering and the Ph.D. degree from the Universitat Politècnica de Catalunya (UPC), Barcelona, Spain, in 2001 and 2006, respectively.

In 2001, he was with the Institute of Geomatics, Spain, as a Research Assistant designing a subaperture SAR processor. In 2002, he was with the Department of Signal Theory and Communications, UPC, where he worked in the field of airborne repeatpass interferometry and airborne differential SAR interferometry. From December 2002 to August 2006, he was an Assistant Professor with the Department of Telecommunications and Systems Engineering, Universitat Autònoma de Barcelona, Barcelona, Spain. In 2006, he joined the Microwaves and Radar Institute, German Aerospace Center (DLR), Wessling, Germany, where, since August 2009, he is Head of the Multimodal Algorithms Group. His research interests include air- and spaceborne SAR processing, SAR interferometry, differential SAR interferometry, and motion compensation for airborne systems.

Dr. Prats was the recipient of the first prize of the Student Paper Competition of the IEEE International Geoscience and Remote Sensing Symposium 2005 Conference held in Seoul, Korea.

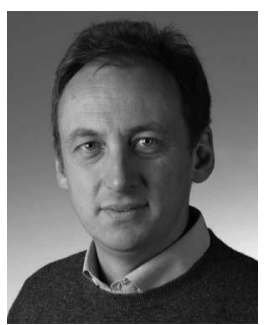

Rolf Scheiber received the Diploma degree from the Technical University of Munich, Munich, Germany, in 1994 and the Ph.D. degree with a thesis on airborne SAR interferometry from the University of Karlsruhe, Karlsruhe, Germany, in 2003, both in electrical engineering.

Since 1994, he has been with the Microwaves and Radar Institute, German Aerospace Center (DLR), Wessling, Germany, where he developed the operational high-precision interferometric SAR processor for its E-SAR airborne sensor. Since 2001, he has been heading the SAR signal processing group within the SAR technology department. His current research interests include algorithm development for high-resolution air- and spaceborne SAR focusing, SAR interferometry, differential SAR interferometry, SAR tomography, as well as radio sounding algorithms and applications.

Dr. Scheiber was awarded as coauthor with the 1996 GRSS Transactions Prize Paper Award for the contribution "Extended Chirp Scaling Algorithm for Air- and Spaceborne SAR Data Processing in Stripmap and ScanSAR Imaging Modes" in 1997.

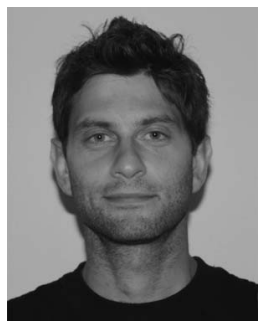

Luca Marotti was born in 1980. He received the degree in electronic engineering from the University of Naples "Federico II," Naples, Italy, in 2006.

In 2005, he joined the SAR technology department of the German Aerospace Centre (DLR), Wessling, Germany, to perform his diploma thesis on detection and polarimetric characterization of coherent scatterers in SAR images. In 2006, he joined again the SAR technology department at DLR where he is currently involved in the realization of a spaceborne SAR interferometric chain and simulation tools for the BIOMASS spaceborne mission, candidate to the European Space Agency Earth Explorer. His main research interest are in the field of SAR processing and SAR interferometry.

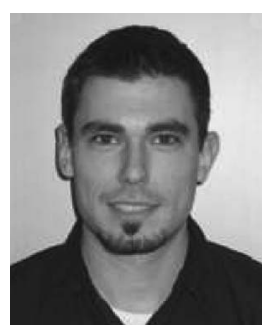

Steffen Wollstadt received the Dipl.-Ing. (M.Sc.) degree in electrical engineering from the Technical University of Darmstadt, Darmstadt, Germany, in 2005, with a thesis on metamaterial-based microstrip antenna design.

In 2006, he joined the Microwaves and Radar Institute, German Aerospace Center (DLR), Wessling, Germany. From 2006 to 2008, he worked in the Satellite SAR Systems Department, Microwaves and Radar Institute, DLR, as Project Engineer in the System Engineering and Calibration part of the TerraSAR-X Ground Segment. He worked on the TerraSAR-X and TanDEM-X missions. Since 2009, he has been with the Radar Concepts Department, Microwaves and Radar Institute, DLR, where he is currently working on Terrain Observation by Progressive Scans Sentinel-1 image quality and synthetic aperture radar performance investigations.

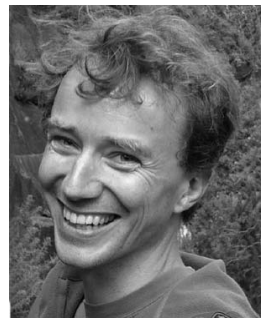

Andreas Reigber (M'02-SM'10) was born in $\mathrm{Mu}$ nich, Germany, in 1970. He received the diploma degree in physics from the University of Constance, Constance, Germany, in 1997 and the Ph.D. degree from the University of Stuttgart, Stuttgart, Germany, in 2001, and the habilitation from the Berlin University of Technology, Berlin, Germany, in 2008.

From 1996 to 2000, he has been with the Microwave and Radar Institute, German Aerospace Center (DLR), Wessling, Germany, working in the field of polarimetric SAR tomography. In 2001, he joined the Antenna, Radar, and Telecom laboratories of the University of Rennes 1, Rennes, France, for a postdoc on radar polarimetry and polarimetric interferometry. From 2002 to 2007, he has been Research Associate at the Computer Vision and Remote Sensing laboratories of the Berlin University of Technology. Since 2008, he is back at the DLR Microwave and Radar Institute, where he is currently the Head of the SAR technology department and directing the airborne SAR activities of the institute. His current main research interests are the various aspects of multimodal SAR, like SAR interferometry, SAR polarimetry, SAR tomography, and time-frequency analyses, as well as filtering and classification aspects of high-resolution SAR data.

Dr. Reigber has received the EUSAR 2000 Student Prize Paper Award for an article on SAR remote sensing of forests, the IEEE TGRS Transactions Prize Paper Award in 2001 for a work on polarimetric SAR tomography as well as the IEEE TGRS Letters Prize Paper Award in 2006 for a work on multipass SAR processing. 